\title{
Reliable and Restricted Quickest Path Problems
}

\author{
Stefan Ruzika* and Markus Thiemann* \\ University of Kaiserslautern \\ Paul-Ehrlich-Straße 14, 67663 Kaiserslautern, Germany. \\ \{ruzika, thiemann\}@mathematik.uni-kl.de
}

February 16, 2011

\begin{abstract}
In a dynamic network, the quickest path problem asks for a path minimizing the time needed to send a given amount of flow from source to sink along this path. In practical settings, for example in evacuation or transportation planning, the reliability of network arcs depends on the specific scenario of interest. In this circumstance, the question of finding a quickest path among all those having at least a desired path reliability arises. In this article, this reliable quickest path problem is solved by transforming it to the restricted quickest path problem. In the latter, each arc is associated a nonnegative cost value and the goal is to find a quickest path among those not exceeding a predefined budget with respect to the overall (additive) cost value. For both, the restricted and reliable quickest path problem, pseudopolynomial exact algorithms and fully polynomial-time approximation schemes are proposed.
\end{abstract}

\section{Introduction}

In dynamic networks, flow units take time to traverse an arc and, there, the quickest path problem generalizes the shortest path problem. Given an amount of flow $U$ and two nodes $s$ and $t$, the goal of the quickest path problem is to find an $s$-t-path with minimum transmission time, that is the total travel time from $s$ to $t$ of this path plus the number of repetitions to send all $U$ flow units along this path (cf. Chen and Chin (1990)). The quickest path problem appears in communication networks, transportation networks, and evacuation modeling (see, among others, (Clímaco et al., 2007; Hamacher and Tjandra, 2002; Moore, 1976)). Polynomial time solution

*Partially supported by BMBF, Project REPKA, FKZ 13N9961 (TU KL). 
algorithms were established for this problem by reducing it to the shortest path problem in a modified network (Chen and Chin, 1990; Rosen et al., 1991).

Numerous variants and extensions of the quickest path problem have been considered, including constrained quickest path problems (Chen, 1994), robust quickest path problems (Ruzika and Thiemann, 2010), and extensions of the quickest path problem to a stochastic-flow network (Lin, 2003).

In practice, operability of arcs in the network may be subject to their reliability, i.e. the probability not to fail. To have a calculable probability of a path's functioning, it is of interest to comprise path reliability in the quickest path problem. Quickest paths with reliabilities have been considered by Xue (1998) and Bang et al. (2003). There, a most reliable quickest path and a quickest most reliable path is sought which is to find a quickest path among the most reliable ones in the first case and a most reliable among the quickest paths in the latter case. In contrast, the reliable quickest path problem considered in this article at hand is understood as finding a quickest path among all paths with at least a desired path reliability predefined by a decision maker. This problem generalizes the most reliable quickest path problem, since it does not require the path to have the best possible reliability.

If usage of an arc is associated with costs and a budget that is not to be exceeded is given, the restricted quickest path problem is of interest. This problem seeks for a quickest path among those paths which obey the budget constraint. It is a generalization of the restricted shortest path problem which has attracted great attention in the literature (Hassin, 1992; Lorenz and Raz, 2001). Bang et al. (2004) considered the related Minimum Cost Quickest Path Problem with Multiple Delay Bounds where a minimum cost path among those paths not exceeding a given transmission time has to be found.

This article is subsequently organized as follows. The next section introduces the quickest path problem, defines its reliable and restricted variants and depicts the equivalence of the two problems. In Section 3, the restricted quickest path problem is solved with a pseudopolynomial algorithm and approximated polynomially. Identical results are deduced for the reliable quickest path problem. The last section gives a conclusion of the article.

\section{Problem Definition}

A dynamic network $G=(N, A)$ with node set $N$ and arc set $A$ is equipped with two kinds of parameters: capacities $u_{i j} \in \mathbb{Z}^{+}$and travel times $\tau_{i j} \in \mathbb{Z}_{0}^{+}$ for all $(i, j) \in A$. The former limits the number of flow units that can 
enter arc $(i, j)$ in a single time step, the latter is the time needed for a flow unit to traverse arc $(i, j)$. Let $s, t \in N$ denote the source and sink node, respectively. The number of nodes and arcs is denoted $n$ and $m$, i.e., it is $n=|N|$ and $m=|A|$. Given an initial amount of flow $U \in \mathbb{Z}^{+}$, the Quickest Path Problem seeks for an $s$-t-path $P$ with minimum transmission time $\sigma(P):=\tau(P)+\left\lceil\frac{U}{u(P)}\right\rceil$ where $\tau(P):=\sum_{(i, j) \in P} \tau_{i j}$ and $u(P):=\min _{(i, j) \in P} u_{i j}$ are the travel time and capacity of path $P$, respectively. Let $\mathcal{P}$ refer to the set of all $s$-t-paths in $G$.

Assume that for each $\operatorname{arc}(i, j) \in A$ a reliability $r_{i j} \in(0,1]$ is given, which describes the probability that this arc is operational. For a path $P \in \mathcal{P}, \prod_{(i, j) \in P} r_{i j}$ is called the path reliability and expresses the probability of a path's functioning. Let $R \in(0,1]$ denote the desired minimum path reliability. The Reliable Quickest Path Problem asks for the quickest path that has a path reliability of at least $R$ :

$$
\begin{array}{ll}
\min & \sigma(P) \\
\text { s. t. } & P \in \mathcal{P} \\
& \prod_{(i, j) \in P} r_{i j} \geq R .
\end{array}
$$

Note that the constraint (1) is equivalent to $\sum_{(i, j) \in P} \ln \left(1 / r_{i j}\right) \leq \ln (1 / R)$. Since $x \mapsto \ln (1 / x)$ is a bijective mapping from $(0,1]$ to $[0, \infty)$, the reliable quickest path problem is equivalent to the Restricted Quickest Path Problem, where cost values $c_{i j} \in \mathbb{R}_{0}^{+}$for all arcs $(i, j) \in A$ and a budget $C \in \mathbb{R}_{0}^{+}$are given and the goal is to find a quickest $s$ - $t$-path with cost not exceeding the budget $C$ :

$$
\begin{array}{ll}
\min & \sigma(P) \\
\text { s. t. } & P \in \mathcal{P} \\
& c(P):=\sum_{(i, j) \in P} c_{i j} \leq C .
\end{array}
$$

Solutions of the restricted and reliable quickest path problem are referred to as restricted quickest paths and reliable quickest paths, respectively.

Given $\varepsilon>0$, an $s$-t-path $Q$ is a $(1+\varepsilon)$-approximation of the restricted quickest path $P^{\star}$, if $c(Q) \leq C$ and $\sigma(Q) \leq(1+\varepsilon) \sigma\left(P^{\star}\right)$. Accordingly, $Q$ is a $(1+\varepsilon)$-approximation of the reliable quickest path $\widetilde{P}$, if $\prod_{(i, j) \in Q} r_{i j} \geq$ $R$ and $\sigma(Q) \leq(1+\varepsilon) \sigma(\widetilde{P})$. A minimization problem is said to admit a fully polynomial-time approximation scheme (FPTAS), if there is a $(1+\varepsilon)$ approximation algorithm with running time polynomial in the input size and in $1 / \varepsilon$ for all instances of the problem (cf. (Papadimitriou and Steiglitz, 1982)). 


\section{Algorithms}

The restricted quickest path problem generalizes the restricted shortest path problem: find an $s$-t-path $P$ with minimum travel time $\tau(P)$ in the set of all paths with $\operatorname{costs} c(P)$ at most $C$ (Hassin, 1992). A restricted quickest path for $U=1$ obviously defines a restricted shortest path. Since the restricted shortest path problem is known to be NP-hard (Garey and Johnson, 1979), this applies to the restricted quickest path problem, too. For the restricted shortest path problem on directed acyclic networks, a pseudopolynomial algorithm has been developed by Hassin (1992) who also proposed an FPTAS. Lorenz and Raz (2001) suggested an improved FPTAS for general directed networks with running time $\mathcal{O}\left(\frac{m n^{2}}{\varepsilon} \log \frac{n}{\varepsilon}\right)$.

These algorithms for the restricted shortest path problem require the budget $C$ to be a nonnegative integer. The integer restriction can be avoided by adding a super source $S$ to $N$ and an $\operatorname{artificial} \operatorname{arc}(S, s)$ to $A$ with $\tau_{S s}=0$, $u_{S s}=\infty$ and cost $c_{S s}=C-\lfloor C\rfloor$. Then, a restricted shortest $S$-t-path with budget $\lceil C\rceil$ refers to a solution of the corresponding restricted $s$ - $t$-path problem with budget $C$.

Hassin's exact algorithms for the restricted shortest path problem on directed acyclic networks are only described for travel times and costs both being integral. However, examining Algorithm B in (Hassin, 1992) reveals that this algorithm only operates on the integer valued travel times. Thus, the arc costs are not required to be integral and, hence, the algorithm is capable of computing exact solutions for restricted shortest path problems as considered in this article. The algorithm has computational complexity $\mathcal{O}(m B)$ where $B$ is an upper bound on the optimal value of the restricted shortest path problem (e.g. the sum of all arc travel times). The generalization of this algorithm for arbitrary directed networks runs in $\mathcal{O}(\mathrm{nmB})$ (Lorenz and Raz, 2001).

For $k \geq 0$ let $G(k)$ be the network with arc set $A(k):=\left\{(i, j) \in A: u_{i j} \geq\right.$ $k\}$. For a restricted quickest path problem, the corresponding restricted shortest path problem in $G(k)$ for $k \geq 0$ is defined on the same cost values and budget constraint.

Lemma 1. Let $Q$ be a solution of the restricted quickest path problem. Then, $Q$ solves the corresponding restricted shortest path problem in $G(u(Q))$.

Proof. Let $P$ be a restricted shortest path in $G(u(Q))$. Then, $\tau(P) \leq \tau(Q)$, $u(P) \geq u(Q), c(P) \leq C$, and $c(Q) \leq C$. Since $Q$ is a restricted quickest path, it is

$$
\tau(Q)+\left\lceil\frac{U}{u(Q)}\right\rceil \leq \tau(P)+\left\lceil\frac{U}{u(P)}\right\rceil .
$$


It follows that $\tau(Q) \leq \tau(P)$ and, hence, $Q$ is a restricted shortest path in $G(u(Q))$.

Theorem 1. Let $u_{1}, \ldots, u_{l}$ be the distinct capacities in $G$. For each $u_{j}$, $j=1, \ldots, l$, let $P_{j}$ be a restricted shortest path in $G\left(u_{j}\right)$. Let

$$
P_{k} \in \underset{j=1, \ldots, l}{\operatorname{argmin}} \sigma\left(P_{j}\right) .
$$

Then, $P_{k}$ solves the restricted quickest path problem.

Proof. Let $Q$ be a solution of the restricted quickest path problem. Let $u_{j_{0}}=u(Q)$ and consider the network $G\left(u_{j_{0}}\right)$. According to Lemma $1, Q$ is a restricted shortest path in $G\left(u_{j_{0}}\right)$. Therefore, it is $\tau\left(P_{j_{0}}\right)=\tau(Q)$ and $u\left(P_{j_{0}}\right) \geq u(Q)$. Thus,

$$
\sigma\left(P_{k}\right) \leq \tau\left(P_{j_{0}}\right)+\left\lceil\frac{U}{u\left(P_{j_{0}}\right)}\right\rceil \leq \tau(Q)+\left\lceil\frac{U}{u(Q)}\right\rceil
$$

and, hence, $P_{k}$ is a restricted quickest path.

Corollary 1. The restricted quickest path problem can be solved in $\mathcal{O}\left(\mathrm{nm}^{2} \mathrm{~B}\right)$.

Proof. A restricted quickest path can be found by solving $l$ restricted shortest path problems, see Theorem 1 . Since $l \leq m$, the computational complexity follows directly from that of the restricted shortest path problem.

For $\varepsilon>0$, a $(1+\varepsilon)$-approximation of the restricted shortest path is a path obeying the budget constraints and having a travel time within a factor $(1+\varepsilon)$ of the optimal travel time.

Theorem 2. Let $\varepsilon>0$ and let $u_{1}, \ldots, u_{l}$ be the distinct capacities in $G$. For each $u_{j}, j=1, \ldots, l$ let $P_{j}$ be $a(1+\varepsilon)$-approximation of the restricted shortest path in $G\left(u_{j}\right)$. Let

$$
P_{k} \in \underset{j=1, \ldots, l}{\operatorname{argmin}} \sigma\left(P_{j}\right) .
$$

Then, $P_{k}$ is a $(1+\varepsilon)$ approximation of the restricted quickest path.

Proof. Let $Q$ be a solution of the restricted quickest path problem and let $u_{j_{0}}=u(Q)$. Then, $Q$ is a restricted shortest path in $G\left(u_{j_{0}}\right)$ due to Lemma 
1 and from definition it is $\tau\left(P_{j_{0}}\right) \leq(1+\varepsilon) \tau(Q)$. With $u\left(P_{j_{0}}\right) \geq u(Q)$ it follows that

$$
\tau\left(P_{j_{0}}\right)+\left\lceil\frac{U}{u\left(P_{j_{0}}\right)}\right\rceil \leq(1+\varepsilon) \tau(Q)+\left\lceil\frac{U}{u(Q)}\right\rceil \leq(1+\varepsilon)\left(\tau(Q)+\left\lceil\frac{U}{u(Q)}\right\rceil\right) .
$$

Corollary 2. The restricted quickest path problem admits an FPTAS running in $\mathcal{O}\left(\frac{m^{2} n^{2}}{\varepsilon} \log \frac{n}{\varepsilon}\right)$.

Proof. Using Theorem 2, at most $m(1+\varepsilon)$-approximations of restricted shortest paths have to be computed, each of which takes $\mathcal{O}\left(\frac{m n^{2}}{\varepsilon} \log \frac{n}{\varepsilon}\right)$ (Lorenz and Raz, 2001).

As shown in Section 2, the reliable and restricted quickest path problems are equivalent. Thus, the reliable quickest path problem is also NPhard. Further, the results on pseudopolynomial and approximation algorithms arise from the corresponding results for the restricted quickest path problem.

Corollary 3. The reliable quickest path problem can be solved in $\mathcal{O}\left(n m^{2} B\right)$. Moreover, the reliable quickest path problem admits an FPTAS running in $\mathcal{O}\left(\frac{m^{2} n^{2}}{\varepsilon} \log \frac{n}{\varepsilon}\right)$.

\section{Conclusion}

Two variants of the quickest path problem are investigated. The reliable quickest path problem is of interest in dynamic networks where arcs may have a probability of failure. The goal of this problem is to find a quickest path among those having at least a desired path reliability predefined by a decision maker. With a parameter transformation, it is shown that the reliable quickest path problem is equivalent to the restricted quickest path problem, where cost values are given for all arcs and the goal is to find a quickest path among those not exceeding a predefined budget. A pseudopolynomial exact algorithm and an FPTAS are proposed for both problems. Since the problems are NP-hard, the presented algorithms are the best achievable. 


\section{References}

Y.-C. Bang, H. Choo, and Y. Mun. Reliability problem on all pairs quickest paths. In ICCS'03: Proceedings of the 2003 International Conference on Computational Science, pages 518-523. Springer, Berlin, 2003.

Y.-C. Bang, I. Hong, S. Lee, and B. Ahn. On algorithms for minimum-cost quickest paths with multiple delay-bounds. In A. Lagan, M. L. Gavrilova, V. Kumar, Y. Mun, C. J. K. Tan, and O. Gervasi, editors, Computational Science and Its Applications ICCSA 2004, volume 3043 of Lecture Notes in Comput. Sci., pages 1125-1133. Springer, Berlin, 2004.

Y.L. Chen. Finding the $\mathrm{k}$ quickest simple paths in a network. Inform. Process. Lett., 50(2):89-92, 1994.

Y.L. Chen and Y.H. Chin. The quickest path problem. Comput. Oper. Res., 17(2):153-161, 1990.

J.C.N. Clímaco, M.M.B. Pascoal, J.M.F. Craveirinha, and M.E.V. Captivo. Internet packet routing: Application of a K-quickest path algorithm. European J. Oper. Res., 181(3):1045-1054, 2007.

M.R. Garey and D.S. Johnson. Computers and Intractability. A Guide to the Theory of NP-Completeness. A Series of Books in the Mathematical Sciences. W.H. Freeman, San Francisco, 1979.

H.W. Hamacher and S.A. Tjandra. Mathematical modelling of evacuation problems-a state of the art. In M. Schreckenberger and S.D. Sharma, editors, Pedestrian and Evacuation Dynamics, pages 227-266. Springer, Berlin, 2002.

R. Hassin. Approximation schemes for the restricted shortest path problem. Math. Oper. Res., 17(1):36-42, 1992.

Y.K. Lin. Extend the quickest path problem to the system reliability evaluation for a stochastic-flow network. Comput. Oper. Res., 30(4):567-575, 2003.

D.H. Lorenz and D. Raz. A simple efficient approximation scheme for the restricted shortest path problem. Oper. Res. Lett., 28(5):213-219, 2001.

M.H. Moore. On the fastest route for convoy-type traffic in flowrateconstrained networks. Transport. Sci., 10(2):113-124, 1976.

C.H. Papadimitriou and K. Steiglitz. Combinatorial Optimization: Algorithms and Complexity. Prentice-Hall, Englewood Cliffs, 1982. 
J.B. Rosen, S.Z. Sun, and G.L. Xue. Algorithms for the quickest path problem and the enumeration of quickest paths. Comput. Oper. Res., 18 (6):579-584, 1991.

S. Ruzika and M. Thiemann. Min-max quickest path problems. Technical report, University of Kaiserslautern, Department of Mathematics, Report in Wirtschaftsmathematik Nr. 130, 2010.

G. Xue. End-to-end data paths: quickest or most reliable? IEEE Commun. Lett., 2(6):156-158, 1998. 